Research Article

\title{
Early Warning for the Construction Safety Risk of Bridge Projects Using a RS-SSA-LSSVM Model
}

\author{
Gang Li $\mathbb{D},{ }^{1}$ Ruijiang Ran $\mathbb{D},{ }^{2}$ Jun Fang $\mathbb{D},{ }^{2}$ Hao Peng $\mathbb{D},{ }^{1}$ and Shengmin Wang $\mathbb{D}^{3}$ \\ ${ }^{1}$ School of Architectural Engineering, Xinyang Vocational and Technical College, Xinyang 464000, China \\ ${ }^{2}$ School of Civil Engineering and Architecture, Wuhan University of Technology, Wuhan 430070, China \\ ${ }^{3}$ School of Safety Science and Emergency Management, Wuhan University of Technology, Wuhan 430070, China \\ Correspondence should be addressed to Shengmin Wang; wsm910429@whut.edu.cn
}

Received 31 August 2021; Revised 1 October 2021; Accepted 23 October 2021; Published 5 November 2021

Academic Editor: Afaq Ahmad

Copyright ( ) 2021 Gang Li et al. This is an open access article distributed under the Creative Commons Attribution License, which permits unrestricted use, distribution, and reproduction in any medium, provided the original work is properly cited.

\begin{abstract}
Bridge engineering is an important component of the transportation system, and early warnings of construction safety risks are crucial for bridge engineering construction safety. To solve the challenges faced by early warnings risk and the low early warning accuracy in bridge construction safety, this study proposed a new early-warning model for bridge construction safety risk. The proposed model integrates a rough set (RS), the sparrow search algorithm (SSA), and the least squares support vector machine (LSSVM). In particular, the initial early warning factors of bridge construction safety risk from five factors (men, machines, methods, materials, and environment) were selected, and the RS was used to reduce the attributes of 20 initial early warning factors to obtain the optimized early warning factor set. This overcame the problem of multiple early warning factors and reduced the complexity of the subsequent prediction model. Then, the LSSVM with the strongest nonlinear modelling ability was selected to build the bridge construction early-warning model and adopted the SSA to optimize the LSSVM parameter combination, improving the early warning accuracy. The Longlingshan Project in Wuhan and the Shihe Bridge Project in Xinyang, China, were then selected as case studies for empirical research. Results demonstrated a significant improvement in the performance of the early-warning model following the removal of redundancy or interference factors via the RS. Compared with the standard LSSVM, Back Propagation Neural Network and other traditional early-warning models, the proposed model exhibited higher computational efficiency and a better early warning performance. The research presented in this article has important theoretical and practical significance for the improvement of the early warning management of bridge construction safety risks.
\end{abstract}

\section{Introduction}

Developing countries such as China are currently implementing large-scale bridge projects [1]. However, these projects require large project investment, complex technology, and extensive contents and are typically established in poor construction environments. This results inextremely high construction safety, with an enhanced probability of serious accidents. In recent years, bridge construction safety accidents (e.g., the impact of the cofferdam, the collapse of floating cranes, and flooding) have been a common occurrence [2]. The early warning of construction safety risk generally involves the monitoring, evaluation, and prediction of factors corresponding to construction safety risk, with the aims of predicting future risks, determining the potential time range of risks, and measuring the strength of risks and their damage degree. Such a system aids decisionmakers in taking appropriate risk control measures [3]. Therefore, the systematic identification, estimation, and early warning control of bridge construction safety risks can effectively reduce construction safety risks and achieve the management goals of bridges while complying with safety regulations.

Numerous scholars have performed in-depth studies on the early warning of risk in many fields. To effectively reduce the financial risks of non-life-insurance enterprises, Yan et al. [4] constructed a financial risk early-warning model. Zhang [5] developed a safety risk early-warning model of the food industry chain, revealing that introducing early warning theory into the field of food safety risk can 
effectively improve the level of risk management. In order to reduce the loss of ship collision, Cheng et al. [6] introduced risk early warning theory into ship collision risk management. Based on climatology, disaster science, and environmental science, Zhang et al. [7] determined that the risk early warning theory should be introduced into the early management of the drought disaster risk of crops such as corn. Sattlee et al. [8] integrated risk early warning theory into the reliability analysis of geological disasters to effectively reduce the losses caused by landslides and falling rocks. Great achievements have been made in the theory of early warning risk, but the research results on early warning of bridge construction safety risk are rarely reported. The early warning of risk denotes the prediction or classification of the target value of research objects. Scholars have adopted various research methods to build risk early-warning models across different fields of research. Ding [9] comprehensively combined the analytic hierarchy process (AHP) and the fuzzy comprehensive evaluation method to construct the risk early-warning model of financial enterprises. However, the fuzzy comprehensive evaluation is a linear weighted evaluation method and cannot effectively reflect the elevated influence of all evaluation indicators. Nonlinear characteristics are also unable to meet the requirements of practical evaluations. The AHP is associated with several disadvantages, such as strong subjectivity and the sensitivity to extreme expert opinions. Based on the strong self-learning ability and nonlinear processing of artificial neural networks (ANNs), Yan et al. [10] constructed an early-warning model of human resource management risk based on the back propagation neural network (BPNN). However, the application of the BPNN in early warning risk research results in several shortcomings, including overfitting, slow convergence, and easy to fall into a local minimum. Wang et al. [11] employed the grey model (GM) to construct an early warning safety risk model of a railway service system. Although the GM is simple to operate, it requires that variables satisfy the multivariate normal distribution, which is difficult to meet in practical applications. Chen and Zhang [12] used the logistic regression model to construct a logistics-based early warning risk management system for the default risk of cultural creative crowdfunding projects. However, the calculation of the logistic regression model is approximate and thus has several shortcomings, such as complex calculations and low prediction accuracy.

The least square support vector machine (LSSVM) inherits the structural risk concept and kernel mapping concept of the standard support vector machine (SVM). Starting from the loss function of machine learning, two norms are used in the objective function of the optimization problem in the LSSVM, and equality constraint is used instead of inequality constraint in SVM standard algorithm, which makes the solution of optimization problem of LSSVM method become a set of linear equations obtained by Kuhn-Tucker condition. The LSSVM trains the SVM by solving the transformed linear equations, which greatly improves the training efficiency of the SVM [13]. In recent years, the LSSVM has been widely used in data prediction, data classification, and other research fields. Zhao et al. [13] used the LSSVM to effectively diagnose aircraft engine faults. Ahmadi et al. [14] successfully simulated the vaporization enthalpies of pure hydrocarbons and petroleum fractions via the LSSVM. Statistical results determined the LSSVM-predicted average relative deviation and $R^{2}$ of the vaporization enthalpies as $0.51 \%$ and 0.9998 , respectively, indicating the high prediction accuracy of the LSSVM. To improve the prediction accuracy of the cotton fabric $K / S$ value, Yu et al. [15] combined particle swarm optimization (PSO) with the LSSVM to construct a new prediction model. In order to overcome the low early warning accuracy, in the current study, the LSSVM is employed to build an early-warning model of bridge construction safety risk.

Despite its strong robustness and generalization ability, the LSSVM cannot simplify the dimensions of information space. For high dimensions or large training samples, the LSSVM often faces problems, such as dimension disaster, and an increase in time consumption due to the limited memory capacity or complex network structure. The rough set (RS) does not require any prior knowledge and removes redundant data without affecting the classification accuracy. It is widely used in the fields of attribute sets [16] and key indicator screening. Introducing the RS into the LSSVM can effectively determine key attributes and reduce the adverse effects of redundancy and multicollinearity among various input variables on prediction accuracy. Therefore, when developing the proposed early-warning model based on the LSSVM, the RS is introduced to solve the problem of multiple early warning factors.

As a new machine learning method, the prediction accuracy and computational performance of the LSSVM depend on the reasonable selection of regularization and kernel width parameters. In the development of a landslide displacement prediction model for rainfall, Zhu et al. [17] employed the genetic algorithm (GA) to determine the LSSVM optimal parameters. However, the GA is associated with several bottlenecks, including complex coding, a slow calculation speed, and easy premature convergence [18]. To improve the accuracy of the LSSVM in predicting concrete strength, Xue [19] optimized the calculation parameters via PSO. However, the PSO is also prone to premature convergence (particularly when dealing with complex multimodal search problems) and has a poor local optimization ability [14].

The sparrow search algorithm (SSA) is a new swarm intelligence optimization approach that was inspired by the foraging and antipredation behaviours of sparrows [20]. When predicting the deboning strain of fibre reinforced polymer reinforced concrete, Li et al. [21] employed the SSA to optimize the initial weight and threshold of the BPNN. Empirical results revealed that the SSA-optimized BPNN surpassed the traditional version in terms of prediction accuracy and robustness. Liu and Rodriguez [22] used the SSA to accurately solve the problem of sustainable energy optimization in residential engineering, a complex and multiobjective nonlinear optimization problem. Wumaier et al. [23] adopted the SSA to optimize the SVM parameter combination, and based on wind turbine fault diagnosis data, the SSA-SVM was clearly superior to GA-SVM and 
PSO-SVM in terms of computing performance. Therefore, the SSA in the proposed method was adopted to determine the LSSVM optimal parameter for efficient computing performance.

Based on the above analysis and review of the relevant literature, a hybrid early-warning model of bridge construction safety risk was developed based on the RS, SSA, and LSSVM. The contributions and innovations of this article are as follows. (1) At present, researches on the early warning of risk mainly focuses on the financial risk, the food safety, the disaster risk, or other fields. In this study, early warning for the construction safety risk of bridge projects was studied, and a detailed case study was made. This provided new insights for early warning and management of bridge construction project. (2) From five aspects (men, machines, materials, methods, and environment), the index system of early warning for construction safety risk of bridge projects was constructed completely, which provided reference and foundation for similar researches. (3) In this study, a novel early-warning model based on the RS, SSA, and LSSVM was constructed, which not only effectively solved the problem of multiple risk early warning factors but also overcame the problem of low precision of traditional early warning methods.

The remaining sections of this article are arranged as follows. In Section 2, the early warning indicator system of bridge construction safety risk and the early-warning model are constructed. In Section 3, the Longlingshan Bridge Project in Wuhan and the Shihe Bridge Project in Xinyang, China, are used to perform the empirical research. Section 4 analyses the computational performance of the earlywarning model proposed in this study, whereas Section 5 summarizes the main conclusions and limitations of this study.

\section{Materials and Methods}

\subsection{Establishment of an Early-Warning Indicator System for Bridge Construction Safety Risk}

2.1.1. Preliminary Selection of Early-Warning Indicators for Bridge Construction Safety Risks. Risk early warning mainly includes three parts: identification, division, and prediction of risk levels. The early-warning management system of construction safety risk in a bridge project plays a key role in scientifically creating an early warning indicator system for safety risk. Referring to the underlying reasons of risk loss during bridge construction, the early warning indicators of bridge construction safety risk was screened from five aspects (men, machines, materials, methods and environment; $4 \mathrm{M} 1 \mathrm{E})[24,25]$, as described in the following.

(1) Men. This includes all participants in the bridge construction process. The construction and management of bridge engineering is generally performed by employees, and thus, their safety has a great influence on bridge construction safety. The illegal operation of workers is a common instigator of construction safety accidents [2]. In general, the more workers violating regulations, the greater the possibility of construction safety accidents and the more serious the consequences. Therefore, the more skilled workers, the lower the safety risks. The safety skills of project managers also play a key role in the daily risk management of construction safety. The stronger the safety skills of project managers, the more effective the daily risk management is. When safety accidents occur, project managers with a good emergency handling ability can effectively reduce secondary accidents and losses caused by safety accidents.

(2) Machines. This includes all machinery and equipment used in the construction project. The safety state of machinery in bridge construction projects is a key influencing factor of construction safety. Mechanical quality, mechanical installation, mechanical operation, and mechanical operation failure are generally the most important factors affecting the safety status of objects in bridge construction [3].

(3) Materials. This includes all materials employed in the bridge construction process. The quality and management problems of materials will consequently result in the quality and safety problems of the whole bridge construction. The most important building materials for bridge construction are concrete and steel, which are the main stress parts of the bridge structure, and they have obvious influence on the structural performance. When the structural performance of bridges is insufficient, safety accidents such as local collapse are easy to occur. Therefore, the qualification rate of these two building materials has a great influence on the construction safety and engineering quality. In addition, related management problems (e.g., stacking and random inspection of materials) may also result in bridge construction safety accidents $[2,24]$.

(4) Methods. This denotes the overall management process of bridge construction. Method indicators related to construction safety include all rules and regulations, technical requirements, organization and management, and construction methods. Combined with the construction technology and characteristics of bridge engineering, the most important indicators are the monitoring and calculation methods, as well as technical disclosure. Furthermore, a large number of new technologies and processes are often used in bridge construction; thus, the proportion of new construction schemes also has a significant impact on construction safety [25]. In general, the greater the proportion of new construction schemes and the less mature the technology, the greater the risk of construction safety.

(5) Environment. This denotes the natural environment and social environment during bridge construction. The characteristics of bridge construction sites and technology and economy lead to a complex construction environment, and there are many natural and social factors that affect bridge construction. Natural disasters, such as earthquakes, typhoons, and rainstorms, will seriously affect construction safety. In bridge construction, the wind has a great influence on bridge construction safety [3]. The social environment of bridge construction mainly refers to the sustainability and socioeconomic stability of engineering projects. 
Table 1 provides details of the five early warning indicators. Here, the qualitative indicator refers to the data obtained by expert investigations, whereas the quantitative indicator is obtained by field investigations, consulting design data, or calculations based on national standards. The benefit indicator increases with the bridge construction safety risk level, whereas the opposite is true for the cost indicator.

\subsubsection{Threshold Value of Early Warning Bridge Construction} Safety Risk. The early-warning threshold of bridge construction safety risk is divided into a general indicator earlywarning threshold and a subindicator early-warning threshold. In the actual construction process, rapidly obtaining quantitative data of risk indicators proves to be a difficult task, particularly as indicators related to early warnings still rely on the observation and subjective judgment of site managers. Therefore, a qualitative and quantitative combination, with a qualitative expert determination, method was adopted to judge the warning situation of early warning indicators. Table 2 reports the division of each indicator and the corresponding descriptions.

The warning degree division of each secondary indicator comprehensively considers the related specifications, such as the Load Code for the Building Structure Design (GB 500092012), the Specifications for Long-Span Highway Bridge Construction Surveys (JTG/T 365-02-2019), the Safety Technical Specifications for Highway Engineering Construction, (JTG F90-2015), the Specifications for Bridge Design Wind Speed Calculations (QX/T 438-2018), the Design Code of the Municipal Bridge (CJJ 11-2011), and the results of previous research of early warning systems [26].

2.2. Introduction to the RS. The RS, a data analysis theory proposed by Polish mathematician Pawlak, is able to deal with the uncertainty, incompleteness, and incompatibility between information and knowledge. The RS-based attribute reduction process involves deleting unimportant or irrelevant redundant attributes while maintaining the classification or decisionmaking ability of information systems unchanged and deducing the classification or decision-making of the problem to be solved $[16,17]$. Therefore, theoretically speaking, applying the RS to the intensive prewarning indicators of bridge construction safety risks can reduce the unimportant indicators while maintaining the prewarning ability, thus improving the calculation accuracy of the subsequent risk prewarning model.

In the RS, information systems can be represented by 4 tuples [16]:

$$
S=\left(U, A, V, f_{S}\right),
$$

where $U$ is the domain of discourse; $A=C \cup D=\left\{a_{1}, a_{2}\right.$, $\left.\ldots, a_{n}\right\}$ is a finite set of non-empty attributes, $C \cap D=\varnothing, C$ is a conditional attribute set, and $D$ is a decision attribute set; $V=\cup_{a \varepsilon A} V_{a}$ is the set of attribute values, and $V_{a}$ is the range of attribute $a \in A$. Function $f_{S}$ is used to assign corresponding attribute values to each object attribute in theory.

The support degree of conditional attribute $\mathrm{C}$ to decision attribute $D$ is an important concept of rough set theory [16]:

$$
\gamma c(D)=\frac{\operatorname{POS}_{C}(D)}{|U|},
$$

where $\operatorname{POS}_{C}(D)$ is denoted as the positive domain of $D$ about $C$, which describes all the element sets in $U$ that can be exactly classified into the $U \mid D$ class according to the knowledge of $C$, and $\gamma_{C}(D)$ indicates the ratio of objects that can be classified into the $U \mid D$ decision class under conditional attribute $C$ and expresses the degree of support of the conditional attribute to the decision attribute.

Assume that there exists $q \in C$ and $C \subseteq A$ in information system $S$, if $\operatorname{PCS}_{C}(D) \neq \operatorname{POS}_{(C-q)}(D)$, then it is said that $q$ is a necessary attribute in $C$ and must be kept; otherwise, $q$ is an unnecessary attribute in $C$ and can be deleted [16].

In information system $S, E \subseteq C \subseteq A$ and $E$ are independent and satisfy $\left(\gamma_{E}(D)=\gamma c(D)\right) \wedge\left(\forall E^{\prime} \subset E\right) \Longrightarrow$ $\left(\gamma_{E^{\prime}}(D) \neq \gamma_{C}(D)\right) . E$ is denoted as the reduction of $C$, $\operatorname{Red}(C)$, and the set of essential attributes in $C$ forms the core of $C$, Core $(C)$. If $E$ is independent, it is considered as the minimum set required to maintain the classification ability of universe $U$ [16].

Although the mathematical theory of RS is very complex, the development of Rosetta, which is a computing software integrating rough set principle, facilitates data set classifications and minimum set searches.

2.3. Introduction to the SSA. The SSA is a novel swarm intelligence optimization algorithm [20]. Compared with classical algorithms, such as the PSO, it exhibits a superior computing performance due to the addition of discoverer and early warnings $[21,22]$.

At each SSA, iteration the location update of the discoverer is described as follows [20]:

$$
X_{i, j}^{t+1}= \begin{cases}X_{i, j}^{t} \cdot \exp \left(\frac{-i}{\alpha \cdot \text { iter }_{\max }}\right), & \text { if } R_{2}<S T, \\ X_{i, j}^{t}+Q \cdot L, & \text { if } R_{2} \geq S T,\end{cases}
$$

where $t$ represents the current iteration number, iter ${ }_{\max }$ is a constant indicating the maximum iteration number, $X_{i, j}$ represents the position information of the $i$-th sparrow in the $j$-th dimension, $\alpha \in(0,1]$ is a random number, $R_{2}$ and $S T$ represent the warning and safety values, respectively, $Q$ is a random number that obeys a normal distribution, $L$ represents a $1 \times d$ matrix, and $d$ is the dimensions of the problem to be optimized.

During each iteration, the position update of the enrollee is described as follows [20]:

$$
X_{i, j}^{t+1}= \begin{cases}Q \cdot \exp \left(\frac{X_{\text {worst }}^{t}-X_{i, j}^{t}}{i^{2}}\right), & \text { if } i>\frac{n}{2}, \\ X_{P}^{t+1}+\left|X_{i, j}^{t}-X_{P}^{t+1}\right| \cdot A^{+} \cdot L, & \text { if otherwise, }\end{cases}
$$

where $X_{P}$ is the best current position occupied by the discoverer, $X_{\text {worst }}$ is the worst position in the world at present, and 
TABLE 1: Primary early-warning indicators of bridge construction safety risk.

\begin{tabular}{|c|c|c|c|c|}
\hline $\begin{array}{l}\text { Primary } \\
\text { indicator }\end{array}$ & Secondary indicator & Unit & Indicator type & Data collection method \\
\hline \multirow{4}{*}{ A1: Men } & A11: Rate of operation violation & $\%$ & Quantitative benefit indicator & On-site investigation and statistics \\
\hline & $\begin{array}{c}\text { A12: Rate of improper emergency } \\
\text { handling }\end{array}$ & $\%$ & Quantitative benefit indicator & On-site investigation and statistics \\
\hline & $\begin{array}{c}\text { A13: Acceptance rate of worker } \\
\text { technology }\end{array}$ & $\%$ & $\begin{array}{l}\text { Quantitative cost-based } \\
\text { indicators }\end{array}$ & On-site investigation and statistics \\
\hline & A14: Safety skills of managers & - & Qualitative cost indicator & Expert investigation \\
\hline \multirow{4}{*}{ A2: Machines } & $\begin{array}{l}\text { A21: Unqualified rate of mechanical } \\
\text { quality }\end{array}$ & $\%$ & Quantitative benefit indicator & Field test and statistics \\
\hline & $\begin{array}{c}\text { A22: Failure rate of mechanical } \\
\text { installation }\end{array}$ & $\%$ & Quantitative benefit indicator & Field test and statistics \\
\hline & A23: Rate of mechanical operation error & $\%$ & Quantitative benefit indicator & On-site investigation and statistics \\
\hline & A24: Rate of mechanical failure & $\%$ & Quantitative benefit indicator & On-site investigation and statistics \\
\hline \multirow{4}{*}{ A3: Materials } & A31: Qualified rate of concrete & $\%$ & $\begin{array}{l}\text { Quantitative cost-based } \\
\text { indicators }\end{array}$ & Field test and statistics \\
\hline & A32: Qualified rate of steel & $\%$ & $\begin{array}{l}\text { Qualitative cost-based } \\
\text { indicator }\end{array}$ & Field test and statistics \\
\hline & A33: Rationality of material stacking & - & $\begin{array}{l}\text { Qualitative cost-based } \\
\text { indicator }\end{array}$ & Expert investigation \\
\hline & A34: Sampling inspection of materials & - & $\begin{array}{l}\text { Qualitative cost-based } \\
\text { indicator }\end{array}$ & Expert investigation \\
\hline \multirow{4}{*}{ A4: Methods } & A41: Rationality of monitoring method & - & $\begin{array}{l}\text { Qualitative cost-based } \\
\text { indicator }\end{array}$ & Expert investigation \\
\hline & $\begin{array}{c}\text { A42: Advancement of calculation } \\
\text { method }\end{array}$ & - & $\begin{array}{l}\text { Qualitative cost-based } \\
\text { indicator }\end{array}$ & Expert investigation \\
\hline & A43: Rate of technical disclosure & $\%$ & $\begin{array}{l}\text { Quantitative cost-based } \\
\text { indicators }\end{array}$ & On-site investigation and statistics \\
\hline & $\begin{array}{l}\text { A44: Proportion of new construction } \\
\text { scheme }\end{array}$ & $\%$ & Quantitative benefit indicator & On-site investigation and statistics \\
\hline \multirow{4}{*}{$\begin{array}{l}\text { A5: } \\
\text { Environment }\end{array}$} & A51: Disaster frequency & - & Quantitative benefit indicator & $\begin{array}{c}\text { Consult design data, local yearbook, } \\
\text { etc. }\end{array}$ \\
\hline & A52: Wind load grade & - & Quantitative benefit indicator & $\begin{array}{l}\text { Field experiment and reference } \\
\text { specification }\end{array}$ \\
\hline & $\begin{array}{l}\text { A53: Sustainability of engineering } \\
\text { project }\end{array}$ & - & $\begin{array}{l}\text { Qualitative cost-based } \\
\text { indicator }\end{array}$ & Expert investigation \\
\hline & A54: Social and economic sustainability & - & $\begin{array}{l}\text { Qualitative cost-based } \\
\text { indicator }\end{array}$ & Expert investigation \\
\hline
\end{tabular}

TABLE 2: Division of early warning indicators of bridge construction safety risk.

Division of warning

degree

No (I)

Mild (II)

Moderate (III)

Severe (IV)
Descriptive explanation

Very low risk and project managers do not need to take any measures.

Risks are accepted, yet some early warning indicators have a small probability of causing bridge construction safety accidents. Project managers must focus some of their attention of the risks.

High-risk and some early-warning indicators exhibit a certain probability of causing bridge construction safety accidents. Project managers must make initially targeted measures.

Very high-risk and some early warning indicators have a high probability of causing bridge construction safety accidents. Project managers should take effective measures immediately; otherwise, construction safety accidents will easily occur.
$A$ is a $1 \times d$ matrix, in which each element is randomly assigned 1 or -1 .

When aware of the danger, the sparrow population will exhibit antipredation behaviour, which is described as follows [20]:

$$
X_{i, j}^{t+1}= \begin{cases}X_{\text {best }}^{t}+\beta \cdot\left|X_{i, j}^{t}-X_{\text {best }}^{t}\right|, & \text { if } f_{i}>f_{g}, \\ X_{i, j}^{t}+K \cdot \frac{\left|X_{i, j}^{t}-X_{\text {worst }}^{t}\right|}{f_{i}-f_{w}+\varepsilon}, & \text { if } f_{i}=f_{g},\end{cases}
$$


where $X_{\text {best }}$ is the current global optimal position, $\beta$ is the step control parameter, $K$ is a random number, $f_{i}$ is the fitness value of the current sparrows, $f_{g}$ and $f_{w}$ are the best and worst fitness values in the world, respectively, and $\varepsilon$ is the smallest constant required to avoid a zero denominator.

Compared with other classical meta-heuristic optimization algorithms, the SSA algorithm possesses advantages, including good stability, strong global search ability, and minimal parameters. The bionics principle of the SSA algorithm simulates the foraging process of sparrows, which is similar to other classical meta-heuristic optimization algorithms and is based on "discoverer-follower" optimization. However, the SSA algorithm further simulates the detection and warning behaviour of sparrows during the foraging process. Not all sparrows are constantly approaching the current optimal solution in each iteration, yet some sparrows will experience detection and early warning. This mechanism enhances the global search ability and faster optimization speed of the SSA algorithm.

2.4. Introduction to the LSSVM. Assume that there is a training set $\left\{\left(x_{i}, y_{i}\right) \mid i=1,2, \ldots, n\right\}$, where $x_{i} \in R^{d}, y_{i} \in R, d$ is the input space dimension, and $n$ is the number of training samples. $x_{i} \in R^{d}$ is projected into feature space $H$ using nonlinear mapping $\phi$ and constructed in $H$ following the structural risk minimization criterion. This process can be described as the following constrained optimization problem [13]:

$$
\begin{aligned}
& \min \left(\frac{1}{2} w^{2}+C \frac{1}{2} \sum_{i=1}^{n} \xi_{i}^{2}\right) \\
& \text { s.t. } y_{i}=w^{\mathrm{T}} \phi\left(x_{i}\right)+b+\xi_{i},
\end{aligned}
$$

where $C$ is the compromise coefficient between the empirical risk and confidence interval, that is, the regularization parameter, $\xi_{i}$ is the uncorrelated random error, $w$ is the weight variable of the prediction function, $\varphi(x)$ is the nonlinear mapping function, and $b$ is the deviation coefficient.

This constrained optimization problem can be transformed into the following form by the Lagrange method [13]:

$$
L=\frac{1}{2} w^{2}+C \frac{1}{2} \sum_{i=1}^{n} \xi_{i}^{2}-\sum_{i=1}^{n} \alpha_{i}\left(w^{T} \phi\left(x_{i}\right)+b+\xi_{i}-y_{i}\right),
$$

where $\alpha_{i}$ is a Lagrange multiplier.

Using the Karush-Kuhn-Tucker condition, equation (7) can be transformed into equality form, allowing us to obtain the linear model [13] by solving $\alpha_{i}$ and $b$ via the least square method.

$$
y=\sum_{i=1}^{n} \alpha_{i} K\left(x, x_{i}\right)+b
$$

where $K\left(x, x_{i}\right)=\phi(x)^{\mathrm{T}} \phi(x)$ is the kernel function.

The radial basis function (RBF) is selected as the kernel function, namely, $(x, y)=\exp \left(-x-y /\left(2 \sigma^{2}\right)\right)$, where $\sigma^{2}$ is the kernel width parameter reflecting the distribution characteristics of the training samples. Because the RBF kernel function satisfies the Mercer condition, calculating the kernel function in the original input space can replace the inner product operation in the mapping feature space.

To ensure the generalization performance of the LSSVM, it is necessary to optimize the regularization and kernel width parameters to determine the optimal parameter combination. At present, the K-fold calculation method or meta-heuristic optimization algorithm are commonly used to determine the optimal calculation parameters.

2.5. Implementation of the Early-Warning Model. The core concepts of the proposed early-warning model are described as follows: (1) The RS is used as the preprocessing system of the LSSVM, which preprocesses the safety risk data of the bridge construction and eliminates the redundant attributes and conflict attributes, thus simplifying the early-warning model structure of the LSSVM, shortening the training and improving the classification performance. (2) The LSSVM model is optimized via the global optimization ability of the SSA, and the parameter combination with the highest calculation accuracy is obtained to construct the optimal earlywarning model of bridge construction safety risk. Figure 1 presents the architecture diagram of the proposed earlywarning model.

In particular, the key steps of the proposed early-warning model for bridge construction safety risk are described in the following.

Step 1. Training and test sets are built, and the research object is determined. The original engineering data are collected based on the bridge construction safety risk early warning indicator system detailed in Section 2.1. Some samples are randomly selected from the original data as training sets and the remaining data are taken as the test sets.

Step 2. Data preprocessing and attribute set. The original engineering data are normalized as the database of early warning research. On the premise of guaranteeing the original classification ability, the redundant or conflicting attributes in the decision table are eliminated via a rough set theory and the minimum conditional attribute set is obtained.

Step 3. The early-warning model based on the LSSVM is trained and optimized. In particular, the early-warning model and kernel function parameters are initialized, the training sample set is input into the model, and the SSA algorithm is implemented to optimize the calculation parameters. Note that the iterative optimization calculation only occurs in the SSA, not in the LSSVM. The optimal calculation parameters and engineering data are input into the LSSVM to determine the early warning results.

Step 4. Early warning identification. The test sets are input into the optimal LSSVM model for early warning identification to obtain the early warning results of bridge construction safety risks. 


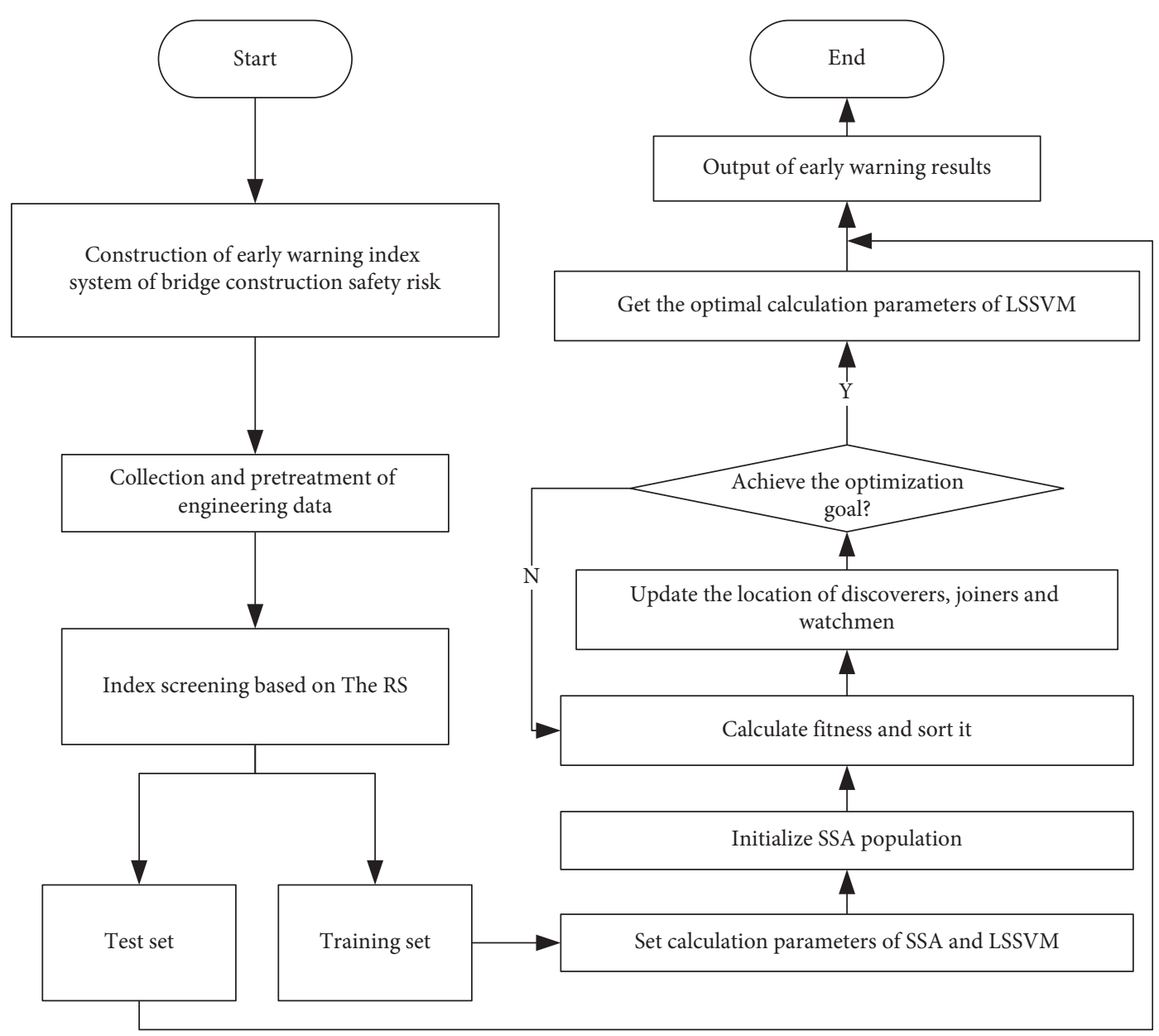

FIgURE 1: Flow chart of the early-warning model proposed in this study.

It should be emphasized that although the three algorithms (RS, SSA, and LSSVM) worked almost independently, these three algorithms belonged to the research framework of bridge construction safety risk early warning. The RS was to solve the problem of many factors affecting early warning and also to simplify the subsequent prediction model. LSSVM optimized by SSA was used to solve the problem of low prediction accuracy. Or it could be considered that both RS and SSA were used to optimize the LSSVM model, in which RS was used to optimize the input variables of LSSVM, and the SSA was used to optimize the optimal calculation parameters of LSSVM.

\section{Case Study}

3.1. Introduction of the Case Study. The Longlingshan Bridge Project in Wuhan and the Shihe Bridge Project in Xinyang, China were selected as the case studies.

The Longlingshan Bridge Project adopts four two-way lanes, with a standard width of $18.5 \mathrm{~m}$ and a total length of $1678.5 \mathrm{~m}$. Its structural form is a restressed concrete continuous beam. The project is located in Wuhan, Hubei Province, China. There are almost no earthquakes, yet heavy rains often occur. Limestone karst caves are located around the project and are generally $0.3-8.1 \mathrm{~m}$ high. The main construction technology of this pile cap is summarized as follows: measure and support the position of the lofting steel sheet, construct the purlin (which also serves as the guide beam), insert the steel sheet pile to the design elevation, and excavate the foundation pit to pour in cushion concrete. Cranes are used as vertical transportation tools during the pier construction process, while scaffolding is set up as an operation platform. The concrete is commercial concrete, and piers lower (higher) than $11 \mathrm{~m}$ are cast by day pump once (twice). The basic construction steps of the concrete box girder are the erection of the supports, the one-time pouring, and overall tensioning. According to the site construction conditions, there are two forms of box girder support: (1) the floor-type bracket with full buckle and (2) the combined bracket (smaller steel pipe bracket for the door opening buckle bracket).

The Shihe Bridge Project in Xinyang is located in Xinyang City, Henan Province, China, with a total length of $203 \mathrm{~m}$. It is a typical thrust concrete-filled steel tube arch bridge with a deck width of $37.5 \mathrm{~m}$ and six lanes in both directions. The construction procedures of the concretefilled steel tubular arch bridge typically include pouring, reinforced, concrete, $\mathrm{V}$-shaped piers and box girders, closing hollow steel tubular arch ribs, installing and tensioning tie bars, pouring concrete into arch ribs, installing and 
tensioning suspenders, hoisting bridge deck beams and longitudinal beams, laying prefabricated hollow slabs on the bridge deck, pouring bridge deck concrete, installing anticollision walls, paving bridge deck asphalt concrete, and so on. The construction of multispan, continuous, concretefilled, steel, tubular arch bridges generally adopts the construction sequence of flowing water from the side span to the middle span. The subsequent construction process can be carried out once the entire bridge is completed. The advantage of this construction scheme is that the uniform loading between spans is realized to the maximum extent, thus effectively ensuring the arch rib line type and increasing the uniformity of the V-shaped buttress and arch.

\subsection{Data Acquisition and Preprocessing}

3.2.1. Data Acquisition and Reliability Analysis. The Longlingshan Bridge and Shihe Bridge Projects were constructed in 700 and 360 days, respectively. 1,060 groups of data from each day during the construction phase of these two projects were collected. Incomplete and questionable data sets were eliminated, and a total of 372 data sets were obtained; 300 (80.64\%) of which were randomly selected as training sets, and the remaining $72(19.36 \%)$ as test sets. Table 3 presents an example of the raw data.

Obtaining engineering data has always been one of the main difficulties in the engineering field. According to different engineering data sources or data acquisition methods, the 20 secondary indicators were roughly divided into the following three categories.

(1) The index data of A51 (Disaster frequency) and A52 (Wind load grade) were obtained by consulting local yearbooks or design data.

Taking Long Lingshan Bridge Project in Wuhan as an example, this study illustrated the data acquisition processes of A51 and A52. According to the statistics of Wuhan Yearbook (http://tjj.wuhan.gov.cn/tjfw/ tjnj/) published by Wuhan Municipal Government, during the period from 2001 to 2020 , there were 47 natural disasters in the urban area of Wuhan, so the score of A51 was 2.35, and 2.35 natural disasters occurred every year on average. The data of A52 was the wind forecast data released by Wuhan Meteorological Bureau (http://hubei.weather.com. $\mathrm{cn} /$ wuhan/index.shtml).

(2) Data of A11 (Rate of operation violation), A12 (Rate of improper emergency handling), A13 (Acceptance rate of worker technology), A21 (Unqualified rate of mechanical quality), A22 (Failure rate of mechanical installation), A23 (Rate of Mechanical operation error), A24 (Rate of mechanical failure), A31 (Qualified rate of concrete), A32 (Qualified rate of steel), A43 (Rate of technical disclosure), and A44 (Proportion of new construction scheme) were obtained by field investigation or field investigation combined with standard calculation. Taking Long Lingshan Bridge Project in Wuhan as an example, this study illustrated the data acquisition process of A11 and A31. Project managers randomly checked the operation of workers in daily inspection. If there were 53 unqualified workers' operations in 125 random checks, the score of A11 should be 0.424 $(0.424=53 / 125)$. Project managers randomly checked the quality of concrete. If 2453 of the 2500 selected times were qualified, the score of A31 should be $98.12(98.12=2453 / 2500 * 100)$. The data of other indicators were also calculated according to the daily management data of the project.

(3) Data of A14 (Safety skills of managers), A33 (Rationality of material stacking), A34 (Sampling inspection of materials), A41 (Rationality of monitoring method), A42 (Advancement of calculation method), A53 (Sustainability of engineering project), and A54 (Social and economic sustainability) were obtained via questionnaires. According to their rich engineering experience, the experts graded the qualitative indicators with reference to Table 2. The average score of 20 experts was taken as the final score of the input variable. To ensure the validity of the expert scoring data, the invited 20 experts had more than 15 years of work experience and were senior engineers. The reliability of the qualitative indicator data is evaluated by determined the Cronbachs' $\alpha$ coefficient (Table 4).

The Cronbachs' $\alpha$ coefficients of all qualitative indicators exceed 0.7 , proving the high reliability of the questionnaire survey results [27]. The acquisition methods of other indicator data are based on field statistics, standard calculations, and official statistics; thus, their reliability is well guaranteed.

3.2.2. Data Preprocessing and Attribute Intensive. To eliminate the influence of dimensional and data-level differences of early warning indicators on the performance of earlywarning models, the data are preprocessed by the normalization methods in equations (9) and (10) for indicators with beneficial type or cost type, respectively:

$$
\begin{aligned}
& x_{i k}^{\prime}=\frac{x_{i \max }-x_{i k}}{x_{i \max }-x_{i \min }}, \\
& x_{i k}^{\prime}=\frac{x_{i k}-x_{i \min }}{x_{i \max }-x_{i \min }},
\end{aligned}
$$

where $x_{\mathrm{i} k}$ and $x_{i k}{ }^{\prime}$ are the $k$-th indicator value of the $i$-th indicator and its normalized value, and $x_{i \text { min }}$ and $x_{i \max }$ are the minimum and maximum values of the $i$-th indicator value, respectively.

Linear correlations may be observed among the $20 \mathrm{sec}-$ ondary indicators. These redundant or interfered secondary indicators are likely to affect the prediction accuracy of the early-warning model. Therefore, this study adopted an attribute reduction algorithm based on the RS. More specifically, redundant irrelevant or unimportant attributes were deleted, and warning indicators that were sensitive to the water state of bridge construction safety risks were identified. 
TABle 3: Original data of the case studies.

\begin{tabular}{|c|c|c|c|c|c|c|c|c|c|}
\hline Secondary indicator & 1 & 2 & 3 & 4 & 5 & 6 & $\cdots$ & 371 & 372 \\
\hline A11 & 0.424 & 1.223 & 0.872 & 0.313 & 2.342 & 4.873 & $\ldots$ & 0.291 & 0.742 \\
\hline A12 & 9.583 & 2.135 & 4.293 & 3.421 & 5.231 & 2.123 & $\cdots$ & 0.234 & 1.882 \\
\hline A13 & 93.21 & 90.31 & 85.74 & 89.07 & 79.42 & 95.25 & $\ldots$ & 76.48 & 89.25 \\
\hline A14 & 86.25 & 72.55 & 83.85 & 94.25 & 69.35 & 86.65 & $\cdots$ & 79.95 & 68.55 \\
\hline A21 & 1.451 & 0.832 & 2.425 & 5.317 & 3.427 & 2.512 & $\cdots$ & 0.982 & 0.428 \\
\hline $\mathrm{A} 22$ & 12.48 & 10.42 & 0.342 & 4.124 & 0.523 & 16.25 & $\ldots$ & 3.411 & 7.234 \\
\hline A23 & 25.25 & 18.14 & 9.413 & 17.94 & 24.31 & 4.510 & $\ldots$ & 7.223 & 5.861 \\
\hline $\mathrm{A} 24$ & 4.112 & 9.379 & 2.345 & 0.512 & 4.294 & 2.512 & $\ldots$ & 0.316 & 3.184 \\
\hline A31 & 98.12 & 97.52 & 98.65 & 99.05 & 96.59 & 94.50 & $\ldots$ & 98.52 & 99.50 \\
\hline A 32 & 99.52 & 98.95 & 99.74 & 99.39 & 99.47 & 97.52 & $\ldots$ & 98.83 & 99.05 \\
\hline A33 & 84.75 & 74.45 & 86.25 & 69.15 & 48.55 & 86.05 & $\ldots$ & 79.55 & 73.45 \\
\hline A34 & 38.55 & 75.25 & 65.85 & 42.45 & 65.25 & 62.85 & $\ldots$ & 58.25 & 79.45 \\
\hline A41 & 67.55 & 74.25 & 85.65 & 75.35 & 79.35 & 78.75 & $\cdots$ & 84.75 & 78.35 \\
\hline A42 & 85.25 & 79.35 & 95.25 & 72.15 & 85.45 & 69.25 & $\ldots$ & 78.45 & 83.65 \\
\hline A43 & 98.41 & 99.58 & 95.25 & 96.23 & 97.14 & 95.42 & $\cdots$ & 99.74 & 98.68 \\
\hline A44 & 25 & 30 & 30 & 45 & 25 & 40 & $\ldots$ & 35 & 20 \\
\hline A51 & 2.35 & 2.35 & 2.35 & 2.35 & 2.35 & 2.520 & $\cdots$ & 2.520 & 2.520 \\
\hline A52 & 5 & 7 & 7 & 5 & 4 & 9 & $\ldots$ & 4 & 6 \\
\hline A53 & 68.35 & 84.25 & 60.55 & 75.35 & 82.45 & 48.95 & $\ldots$ & 68.45 & 84.25 \\
\hline A54 & 75.25 & 90.45 & 84.55 & 78.05 & 68.75 & 25.45 & $\cdots$ & 70.25 & 89.35 \\
\hline Warning degree & I & II & I & II & III & II & $\cdots$ & II & II \\
\hline
\end{tabular}

TABLE 4: Reliability analysis of the qualitative indicators.

\begin{tabular}{lcccc}
\hline Secondary indicator & A14 & A33 & A34 & A41 \\
\hline Cronbachs' $\alpha$ & 0.704 & 0.823 & 0.734 & 0.785 \\
Result & Pass & Pass & Pass & Pass \\
Secondary indicator & A42 & A53 & A54 & - \\
Cronbachs' $\alpha$ & 0.824 & 0.864 & 0.723 & - \\
Result & Pass & Pass & Pass & - \\
\hline
\end{tabular}

The preprocessed 372 data sets were imported into Rosetta, and the attribute reduction was performed via Johnson's algorithm. Table 5 reports the attribute reduction results, namely, the most important indicators based on the RS analysis.

The eight secondary indicators in Table 5 were also identified as the most important indicators affecting the construction safety risks in previous research $[24,25]$. This acts as a validation of our results.

As the RS is a data processing tool, the final RS-based screening of the indicator system may differ for different engineering data sets. Thus, the final early warning indicator system in Table 5 is only applicable to the two engineering cases selected in this study.

\subsection{Early Warning of Bridge Construction Safety Risks}

3.3.1. Optimization of LSSVM Parameters Based on the SSA. The SSA specifications are as follows $[22,23]$ : population size of 100 , maximum iteration number of 200 , safety threshold of 0.7 , discoverers account equal to $30 \%$ of the population size, the maximum permissible error is 0.00001 , and 10 sparrows aware of danger. Calculations were performed in Matlab 2016a (MathWorks) on a computer with the Intel i7 processor configured at $3.40 \mathrm{GHz}$ and a memory of $31 \mathrm{~GB}$. In the LSSVM model, the range of regularization parameters was limited to $[0.01,1000]$, and the range of the kernel function width coefficient was limited to [0.001,10000].

In the iterative optimization process of the SSA, the average value of four warning errors was selected as the fitness function of the SSA. The lower the average value of early warning errors, the lower the function value of fitness function and the better parameter combination.

In order to optimize the LSSVM model parameters for the early warning of the bridge construction safety risks, 170 sets of preprocessed training data were input into the calculation program in Matlab. Figure 2 depicts the adaptive function curve of the SSA. In the enlarged view of Figure 2, the filled rectangular points represented the points with the minimal fitness function.

The fitness function of the SSA is observed to decline rapidly from $44 \%$ to approximately $3 \%$ at the initial stage. Table 6 tracks the optimization calculation process of the SSA algorithm.

The SSA is observed to reach convergence in the 51th generation, converging to $3.657321 \%$. Figure 2 and Table 6 reveal the ability of the SSA to effectively optimize the LSSVM model parameters in the 51th generation. Following the optimization, the optimal parameter combination is determined with kernel function and penalty factor parameters equal to 13.42 and 3.24 , respectively.

3.3.2. Early Warning Calculations. The kernel function and penalty factor parameters (13.42 and 3.24, respectively) were input into the LSSVM model to construct the optimized early-warning model. Table 7 reports the results of the 72 preprocessed sets of test data imported into the optimized LSSVM model.

The parameters results reveal the high calculation accuracy of the proposed prediction model, with a very low misjudgement ratio for different warning degrees. The 
TABLe 5: Final early warning indicator system based on the RS.

\begin{tabular}{lc}
\hline Primary indicators & Secondary indicators \\
\hline A1: Men & A11: Rate of operation violation \\
& A14: Safety skills of managers \\
A2: Machines & A21: Unqualified rate of mechanical quality \\
& A24: Rate of mechanical failure \\
A3: Materials & A31: Qualified rate of concrete \\
A4: Methods & A32: Qualified rate of steel \\
A5: Environment & A44: Proportion of new construction scheme \\
\hline
\end{tabular}

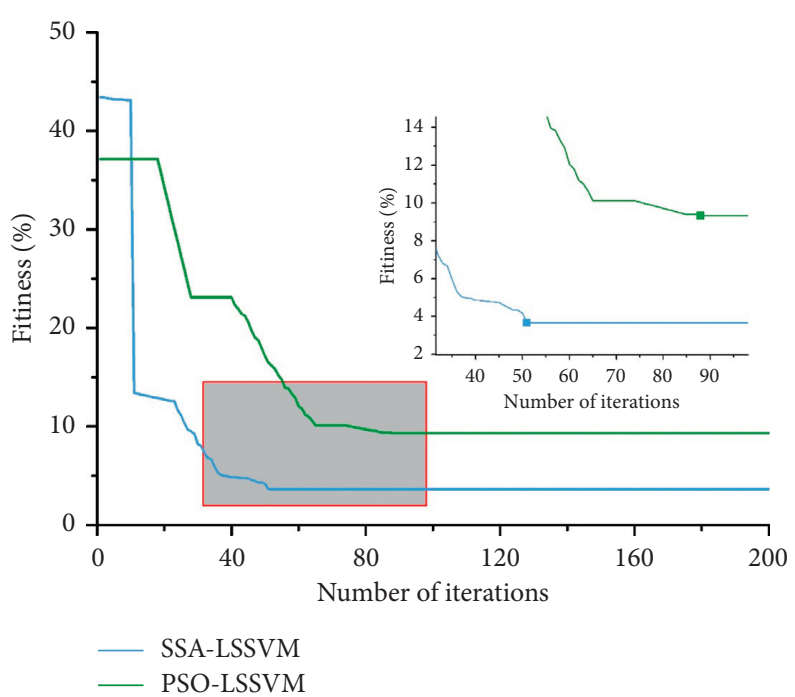

FIgURE 2: Adaptive function curve of different meta-heuristic algorithms.

TABLE 6: Detailed optimization process of the SSA.

\begin{tabular}{lccc}
\hline Iterations & $\begin{array}{c}\text { Fitness } \\
(n-1)\end{array}$ & Fitness $(n)$ & $\begin{array}{c}\text { Fitness }(n)-\text { Fitness } \\
(n-1)\end{array}$ \\
\hline 49 & 4.336809 & 4.332868 & $0.003941001>0.00001$ \\
50 & 4.332868 & 4.166309 & $0.166559012>0.00001$ \\
51 & 4.166309 & 3.657321 & $0.508988>0.00001$ \\
52 & 3.657321 & 3.657321 & $0<0.00001$ \\
200 & 3.657321 & 3.657321 & $0<0.00001$ \\
\hline
\end{tabular}

highest proportion of prediction errors occurred in No (I), which fully met the needs of the engineering practices. For the moderate (III) and severe (IV) cases, two important warning degrees, the proposed prediction model was completely correct. Thus, the proposed model has strong potential in engineering applications.

The proposed model is basedon data; thus, the statistical analysis of the prediction results is required, and the calculations were repeated 1,000 times. Table 8 reports the average of the 1,000 calculations.

The proposed prediction model exhibits a high calculation accuracy for the 1,000 repeated calculations, with extremely low misjudgement ratios for different alarm levels. The highest proportion of prediction errors equals just $1.97 \%$, occurring for no (I). This low error rate fully meets the needs of engineering practices. The high calculation accuracies for the moderate (III) and severe (IV) levels also fully meet the needs of engineering practices. These results further demonstrate the high accuracy of the proposed model and its corresponding potential applicability in the field of engineering.

To further analyse the calculation accuracy of different prediction methods, this study calculated the average value of prediction errors. The calculation results showed that the average prediction error of the prediction model proposed in this study was only $1.025 \%$, which was the lowest among all the results.

Computational time and stability are additional key indicators of the computational ability of prediction models. Table 9 reports the average calculation time of $1,000 \mathrm{cal}-$ culations and the standard deviation of the prediction results.

The running time of our prediction model is observed to be just $3.241 \mathrm{~s}$. Furthermore, the standard deviation of the prediction model results is very low, indicating the high stability of the model.

For this case study, the data structure characteristics of randomly selected training sets may exert a strong influence on the prediction performance of the model. We thus repeated the above calculation 10 times, with the training set randomly selected each time (Table 10).

The difference of the 10 repeated calculations is observed to be minimal, indicating the limited influence of the data structure characteristics from the randomly selected training 
TABLE 7: Results of the early-warning model proposed in this study.

\begin{tabular}{lccc}
\hline Warning degree & Actual warning degree & \multicolumn{2}{c}{ The model proposed in this study } \\
& & Predicted & Rate of prediction errors (\%) \\
\hline I & 27 & 28 & 3.70 \\
II & 34 & 33 & 2.94 \\
III & 8 & 8 & 0.00 \\
IV & 3 & 3 & 0.00 \\
\hline
\end{tabular}

TABLE 8: Average value of the 1000 early warning results of different models.

\begin{tabular}{|c|c|c|c|c|c|c|c|}
\hline Warning degree & Actual & Predicted & Rate of errors (\%) & Predicted & Rate of errors (\%) & Predicted & Rate of errors (\%) \\
\hline & & \multicolumn{2}{|c|}{ The proposed model } & \multicolumn{2}{|c|}{ The proposed model without RS } & \multicolumn{2}{|r|}{ LSSVM } \\
\hline I & 27 & 27.531 & 1.97 & 25.024 & 7.32 & 16.952 & 37.2 \\
\hline II & 34 & 33.524 & 1.40 & 36.084 & 6.13 & 42.084 & 23.8 \\
\hline III & 8 & 7.944 & 0.70 & 7.840 & 2.00 & 8.716 & 8.95 \\
\hline \multirow[t]{2}{*}{ IV } & 3 & 3.001 & 0.03 & 3.052 & 1.73 & 4.248 & 41.6 \\
\hline & & \multicolumn{2}{|r|}{ SSA-BP } & \multicolumn{2}{|c|}{ PSO-LSSVM } & \multicolumn{2}{|r|}{ PSO-BP } \\
\hline I & 27 & 36.723 & 36.0 & 21.414 & 20.7 & 34.482 & 33.5 \\
\hline II & 34 & 26.521 & 22.0 & 36.498 & 7.35 & 26.567 & 21.9 \\
\hline III & 8 & 5.667 & 29.2 & 10.036 & 25.45 & 7.011 & 12.4 \\
\hline IV & 3 & 3.089 & 29.7 & 4.052 & 49.2 & 3.940 & 3.13 \\
\hline
\end{tabular}

TABLE 9: Average calculation time and standard deviation of 1000 calculations.

\begin{tabular}{|c|c|c|c|c|c|c|}
\hline Model & The proposed model & The proposed model without RS & LSSVM & SSA-BPNN & PSO-LSSVM & PSO-BPNN \\
\hline Average calculation time (s) & 3.241 & 3.826 & 0.937 & 4.856 & 4.642 & 5.752 \\
\hline Standard deviation of I & 0.00305 & 0.00524 & 0.02221 & 0.00552 & 0.01245 & 0.01046 \\
\hline Standard deviation of II & 0.00731 & 0.00793 & 0.02597 & 0.00723 & 0.02213 & 0.01475 \\
\hline Standard deviation of III & 0.00645 & 0.00830 & 0.02424 & 0.01420 & 0.04174 & 0.09422 \\
\hline Standard deviation of IV & 0.01023 & 0.01384 & 0.04552 & 0.01525 & 0.01463 & 0.02425 \\
\hline
\end{tabular}

TABLE 10: Prediction accuracy of repeated randomly selected training sets.

\begin{tabular}{|c|c|c|c|c|c|c|c|c|c|c|}
\hline \multirow{2}{*}{ Warning degree } & \multicolumn{10}{|c|}{ Rate of errors (\%) } \\
\hline & $1 \mathrm{st}$ & 2nd & $3 \mathrm{rd}$ & 4 th & 5 th & 6 th & 7 th & 8 th & 9th & 10th \\
\hline I & 1.97 & 1.93 & 1.96 & 2.12 & 1.92 & 1.90 & 2.01 & 1.99 & 1.93 & 1.97 \\
\hline II & 1.40 & 1.39 & 1.39 & 1.41 & 1.39 & 1.41 & 1.40 & 1.38 & 1.40 & 1.30 \\
\hline III & 0.70 & 0.73 & 0.69 & 0.71 & 0.69 & 0.72 & 0.68 & 0.71 & 0.70 & 0.68 \\
\hline IV & 0.03 & 0.04 & 0.03 & 0.04 & 0.02 & 0.03 & 0.05 & 0.03 & 0.03 & 0.03 \\
\hline
\end{tabular}

sets of the case study on the model prediction performance. The maximum prediction error is $2.12 \%$, fully meeting the needs of engineering practices.

\section{Discussion}

4.1. Comparative Analysis of the Attribute Intensive Calculation Results. Correlation analysis [28] and the mean impact value (MIV) [29] are commonly employed as index screening tools. For the former, the Pearson correlation coefficient $(r)$ is typically used to investigate the linear correlation between different variables [28]:

$$
r=\frac{\sum_{i=1}^{n}\left(x_{i}-\bar{x}\right)\left(y_{i}-\bar{y}\right)}{\sqrt{\sum_{i=1}^{n}\left(x_{i}-\bar{x}\right)^{2}\left(y_{i}-\bar{y}\right)^{2}}}
$$

where $x_{i}$ and $y_{i}$ are the values of the first and second variables, respectively, and $\bar{x}$ and $\bar{y}$ are the average values of the first and second variables, respectively.
The absolute value of $r$ can effectively characterize the linear correlation between variables: $r>0.8$ denotes a strong linear correlation between two variables, $r>0.7$ denotes a moderate correlation, and $r<0.7$ denotes almost no linear correlation. Moreover, $r>0(r<0.8)$ represents a positive (negative) correlation.

The normalized engineering data in Table 3 was taken to calculate the Pearson correlation coefficient between an index and the warning degree on equation (11). This also denotes the correlation coefficient between input and output variables. Table 11 reports the results, where bold values indicate absolute values greater than 0.7.

Alarm degrees A12, A13, A23, A32, A33, A41, A44, and $A 54$ are observed to correlate strongly with the output index. Therefore, the remaining 12 indicators were selected as the key risk early warning indicators and input into the SSA-LSSVM model for subsequent calculations. Table 12 reports the results of the calculations repeated 1,000 times. 
TABle 11: Pearson correlation coefficient between an index and the alarming degree.

\begin{tabular}{|c|c|c|c|c|}
\hline Secondary indicator & A11 & A12 & A13 & A14 \\
\hline Correlation coefficient & 0.3144 & 0.7812 & -0.8250 & 0.2275 \\
\hline Secondary indicator & A21 & A22 & A23 & A24 \\
\hline Correlation coefficient & 0.7424 & 0.5384 & 0.8462 & -0.6380 \\
\hline Secondary indicator & A31 & A32 & A33 & A34 \\
\hline Correlation coefficient & 0.3127 & -0.7263 & 0.7422 & -0.0125 \\
\hline Secondary indicator & $\mathrm{A} 41$ & $\mathrm{~A} 42$ & $\mathrm{~A} 43$ & A44 \\
\hline Correlation coefficient & 0.7423 & -0.4352 & -0.2354 & 0.7421 \\
\hline Secondary indicator & A51 & A52 & A53 & A54 \\
\hline Correlation coefficient & 0.3261 & 0.2742 & -0.4630 & 0.9031 \\
\hline
\end{tabular}

TABLE 12: Results determined from 1,000 repeated calculations of different variable screening methods.

\begin{tabular}{|c|c|c|c|c|c|c|}
\hline \multirow[t]{2}{*}{ Warning degree } & \multicolumn{2}{|c|}{ The proposed model } & \multicolumn{2}{|c|}{$\begin{array}{l}\text { The SSA-LSSVM with the } \\
\text { correlation analysis }\end{array}$} & \multicolumn{2}{|c|}{ The SSA-LSSVM with MIV } \\
\hline & Predicted & Rate of errors (\%) & Predicted & Rate of errors (\%) & Predicted & Rate of errors (\%) \\
\hline I & 27.531 & 1.97 & 29.411 & 8.91 & 26.242 & 2.81 \\
\hline II & 33.524 & 1.40 & 39.036 & 14.8 & 34.654 & 1.92 \\
\hline III & 7.944 & 0.70 & 10.381 & 29.8 & 8.091 & 1.14 \\
\hline IV & 3.001 & 0.03 & 3.172 & 5.73 & 3.013 & 0.43 \\
\hline
\end{tabular}

Following the method of [29], the MIV method was employed to screen out the key risk indicator and input them into the SSA-LSSVM model for subsequent calculations (Table 12).

Compared with the correlation analysis and the MIV method, this study adopted RS to screen key risk indicators and obtained the best calculation accuracy. These results prove the advancement of the RS presented in this study.

\subsection{Comparative Analysis of Different Early-Warning Models.} To demonstrate the advancement of the early-warning model proposed in this study, a comparative analysis was performed based on two factors: the advancement of the optimization algorithm and the advancement of the nonlinear modelling method. The proposed model without the RS, the LSSVM, the SSA-BPNN, the PSO-LSSVM, and the PSO-BPNN were systematically ran. Tables 9 and 10 report the results, respectively. Based on the parameter setting method of [10], the sigmoid activation function was adopted for the BPNN, with a minimum convergence error of $10^{-5}$. For the PSO algorithm, following the parameter setting method of references $[15,30]$, the maximum iteration number was set as 200 , the weight factor varied linearly from 0.4 to 0.9 , and the acceleration constants $\mathrm{C} 1$ and $\mathrm{C} 2$ were both equal to 2 .

4.2.1. Advancement of the Selected Optimization Algorithm. Figure 2 reveals that the fitness function of the SSA converged to approximately $3.5 \%$ in the $60-80$ generation during the LSSVM parameter search, whereas the PSO fitness function converged in 110-120 generations. Thus, the SSA converged faster than the PSO, which is consistent with results from the previous literature $[21,22]$.

Table 9 demonstrates the running time of the proposed prediction model to be $1.401 \mathrm{~s}$ lower than that of the PSOLSSVM. More specifically, for the typical nonlinear optimization problem of optimizing the LSSVM parameters, the SSA exhibited a better global fast retrieval ability than the PSO. Comparing the average running times of the PSOBPNN and SSA-BPNN, the SSA algorithm revealed to save $0.896 \mathrm{~s}$. The SSA exhibited an improved global fast retrieval ability in searching for the optimal initial threshold and initializing the BPNN.

These results prove the superior ability of the SSA algorithm for global fast retrievals.

\subsubsection{Advancement of the Selected Nonlinear Modelling} Method. Table 9 demonstrates the running time of the proposed prediction model to be $1.625 \mathrm{~s}, 1.401 \mathrm{~s}$, and $2.511 \mathrm{~s}$ lower than that of SSA-BPNN, PSO-LSSVM, and PSOBPNN, respectively. This highlights the global fast retrieval ability of the SSA and the simple structure of the LSSVM.

The running time of the proposed prediction model is clearly superior to that without the RS. This can be attributed to the removed redundant attributes of the original data from the attribute reduction of the RS. The reduced minimum conditional attribute set was consequently more representative and reduced the complexity of the LSSVM model. Note that the LSSVM exhibited the shortest running time because it does not involve iterative optimization calculations.

The LSSVM exhibited an improved calculation accuracy and stability compared with the BPNN (Tables 9 and 10). This is linked to the distinct calculation principles of the two methods. The LSSVM class prediction method determines the segmentation hyperplane with less support vectors, whereas the neural network-based prediction method follows the law of large numbers in nonlinear modelling. More specifically, the more training data samples, the higher the accuracy of the prediction results, with sample sets often required to be more than ten times the number of input variables. Therefore, under sufficient historical data and 
large research samples, the neural network-based prediction method has an advantage over LSSVM prediction methods in terms of nonlinear modelling ability and computational performance. However, for insufficient historical data and small research samples, the nonlinear modelling ability and computational performance of the LSSVM prediction methods are significantly superior to those based on artificial neural networks.

\section{Conclusions}

In the current study, an early-warning model of bridge engineering construction safety risk based on the RS, SSA, and LSSVM was constructed. The RS was used to effectively solve the problem of multiple risk early warning factors, whereas the LSSVM model optimized via the SSA overcame the low precision of traditional early warning methods. The construction safety risks of two typical bridge projects in China were predicted. Results demonstrated the warning error of the proposed warning model for bridge construction safety risk to be low for different warning levels. In addition, the number of misjudged samples was less than or equal to one. Compared with the LSSVM, SSA-BP, PSO-LSSVM, PSO-BP, and other models, the proposed model exhibits a fast calculation speed, high early warning accuracy, and strong robustness.

In addition, by employing $4 \mathrm{M} 1 \mathrm{E}$, the early warning index system of bridge construction safety risk was constructed, including 20 secondary indexes. Based on the engineering data of the research object, the index system was screened by the RS, removing the redundant or unrealistic attributes of the original data and effectively improving the early warning accuracy of the LSSVM model. For the restressed concrete continuous beam project and concretefilled steel tubular arch bridge selected in this study, the operation violation rate, management safety skills, unqualified rate of mechanical quality, mechanical failure rate, concrete qualified rate, steel qualified rate, promotion of new construction schemes, and the wind load grade were the most important risk warning indicators.

Despite the substantial advancement made by this study in the research field, our proposed model faces several limitations. For example, just two case studies (the Longlingshan Bridge Project in Wuhan and the Shihe Bridge Project in Xinyang) were selected. However, bridges can take the form of numerous structural types and construction techniques. Further research will apply the research results of this study to additional bridges with different structural types or construction techniques. Moreover, the expert experience method, which is typically subjective, was adopted to determine the warning limit of early warnings for bridge construction safety risk. Although the conclusion of the case study was consistent with the actual situation, the determination of the early warning threshold requires further quantification. Lastly, the classification accuracy and performance of the LSSVM depend on the calibration of its parameters. Future work will focus on exploring effective multiobjective optimization techniques to optimize the LSSVM parameter combination and improve its prediction performance.

\section{Abbreviations}

AHP: Analytic hierarchy process

ANNs: Artificial neural networks

BPNN: Back propagation neural network

GA: Genetic algorithm

GM: $\quad$ Grey model

LSSVM: Least squares support vector machine

MIV: $\quad$ Mean impact value

PSO: Particle swarm optimization

RS: $\quad$ Rough set

SSA: Sparrow search algorithm

SVM: Support vector machine

4M1E: Men, machines, materials, methods and environment.

\section{Data Availability}

The case analysis data used to support the findings of this study are available from the corresponding author upon request.

\section{Conflicts of Interest}

The authors declare that there are no conflicts of interest regarding the publication of this paper.

\section{Acknowledgments}

This study was supported by the Science and Technology Project of Wuhan Urban and Rural Construction Bureau, China (201943).

\section{References}

[1] S.-y. Song, J. Guo, Q.-k. Su, and G. Liu, "Technical challenges in the construction of bridge-tunnel sea-crossing projects in China," Journal of Zhejiang University - Science, vol. 21, no. 7, pp. 509-513, 2020.

[2] A. P. C. Chan, Y. Yang, and A. Darko, "Construction accidents in a large-scale public infrastructure project: severity and prevention," Journal of Construction Engineering and Management, vol. 144, no. 10, Article ID 05018010, 2018.

[3] S. S. Lin, S. L. Shen, A. Zhou, and Y. S. Xu, "Risk assessment and management of excavation system based on fuzzy set theory and machine learning methods," Automation in Construction, vol. 122, Article ID 103490, 2021.

[4] C. Yan, L. Wang, W. Liu, and M. Qi, "Financial early warning of non-life insurance company based on RBF neural network optimized by genetic algorithm," Concurrency and Computation: Practice and Experience, vol. 30, no. 23, e4343 pages, 2018.

[5] Y. Zhang, "Food safety risk intelligence early warning based on support vector machine," Journal of Intelligent and Fuzzy Systems, vol. 38, no. 6, pp. 6957-6969, 2020.

[6] Z. Y. Cheng, Y. L. Li, and B. Wu, "Early warning method and model of inland ship collision risk based on coordinated collision-avoidance actions," Journal of Advanced Transportation, vol. 2020, Article ID 5271794, 14 pages, 2020.

[7] Q. Zhang, J. Zhang, C. Wang, L. Cui, and D. Yan, "Risk early warning of maize drought disaster in Northwestern Liaoning 
Province, China," Natural Hazards, vol. 72, no. 2, pp. 701-710, 2014.

[8] M. Sattele, M. Krautblatter, M. Brundl, and D. Straub, "Forecasting rock slope failure: how reliable and effective are warning systems?" Landslides, vol. 13, no. 4, pp. 37-750, 2016.

[9] Q. Ding, "Risk early warning management and intelligent real-time system of financial enterprises based on fuzzy theory," Journal of Intelligent and Fuzzy Systems, vol. 40, no. 4, pp. 6017-6027, 2021.

[10] X. Yan, X. W. Deng, and S. H. Sun, "Analysis and simulation of the early warning model for human resource management risk based on the BP Neural Network," Complexity, vol. 2020, Article ID 8838468, 11 pages, 2020.

[11] L. N. Wang, R. F. Mou, X. Z. Yu, and R. Yang, "The design and implementation of security risk warning and prevention system for railway service system," Agro Food Industry HiTech, vol. 28, no. 3, pp. 264-267, 2017.

[12] J. Z. Chen and H. Z. Zhang, "Study on early warning of cultural and creative crowdfunding defaults based on logistic SVM," Journal of Systems Science and Mathematical Sciences, vol. 40, no. 12, pp. 2320-2331, 2020.

[13] Y. P. Zhao, J. J. Wang, X. Y. Li, G. J Peng, and Z Yang, "Extended least squares support vector machine with applications to fault diagnosis of aircraft engine," ISA Transactions, vol. 97, pp. 189-201, 2020.

[14] H. Ahmadi, H. Ahmadi, and A. Baghban, "Modeling vaporization enthalpy of pure hydrocarbons and petroleum fractions using LSSVM approach," Energy Sources, Part A: Recovery, Utilization, and Environmental Effects, vol. 42, no. 5, pp. 569-576, 2020.

[15] C. B. Yu, Z. W. Xi, Y. L. Lu, and K. Tao, "K/S value prediction of cotton fabric using PSO-LSSVM," Textile Research Journal, vol. 90, no. 23-24, pp. 2581-2591, 2020.

[16] H. Cao, "The utilization of rough set theory and data reduction based on artificial intelligence in recommendation system," Soft Computing, vol. 25, no. 3, pp. 2153-2164, 2020.

[17] X. Zhu, S.-q. Ma, Q. Xu, and W.-d. Liu, "A WD-GA-LSSVM model for rainfall-triggered landslide displacement prediction," Journal of Mountain Science, vol. 15, no. 1, pp. 156-166, 2018.

[18] W. Deng, S. F. Shang, X. Cai, and H. Zhao, "Quantum differential evolution with cooperative coevolution framework and hybrid mutation strategy for large scale optimization," Knowledge-Based Systems, vol. 224, Article ID 107080, 2021.

[19] X. H. Xue, "Evaluation of concrete compressive strength based on an improved PSO-LSSVM model," Computers and Concrete, vol. 21, no. 5, pp. 505-511, 2018.

[20] J. Xue and B. Shen, "A novel swarm intelligence optimization approach: sparrow search algorithm," Systems Science \& Control Engineering, vol. 8, no. 1, pp. 22-34, 2020.

[21] G. B. Li, T. Y. Hu, and D. W. Bai, "BP neural network improved by sparrow search algorithm in predicting debonding strain of FRP-Strengthened RC beams," Advances in Civil Engineering, vol. 2021, Article ID 9979028, 13 pages, 2021.

[22] B. Liu and D. Rodriguez, "Renewable energy systems optimization by a new multi-objective optimization technique: a residential building," Journal of Building Engineering, vol. 35, Article ID 102094, 2021.

[23] T. Wumaier, C. Xu, H. Y. Guo, J. Zhijie, and Z. Huajian, "Fault diagnosis of wind turbines based on a support vector machine optimized by the sparrow search algorithm," IEEE Access, vol. 9, pp. 69307-69315, 2021.

[24] H. Zhou, Y. H. Zhao, Q. Shen, Y. Liu, and H. Cai, "Risk assessment and management via multi-source information fusion for undersea tunnel construction," Automation in Construction, vol. 111, Article ID 103050, 2020.

[25] P. Liu, M. C. Xie, J. Bian, and H. Li, "A hybrid PSO-SVM model based on safety risk prediction for the design process in metro station construction," International Journal of Environmental Research and Public Health, vol. 17, no. 5, 1714 pages, 2020.

[26] L. Y. Ding and C. Zhou, "Development of web-based system for safety risk early warning in urban metro construction," Automation in Construction, vol. 34, pp. 45-55, 2013.

[27] L. Su and S. Peng, "Metallogenic correlations for the Fe-Nbree mineralization in the west mine of the bayan obo deposit, inner Mongolia, China," Acta Geologica Sinica - English Edition, vol. 92, no. 2, pp. 614-626, 2018.

[28] $\mathrm{H}$. Wu and J. W. Wang, "A method for prediction of waterlogging economic losses in a subway station project," Mathematics, vol. 9, no. 12, p. 1421, 2021.

[29] M. A. Bujang, E. D. Omar, E. D. Omar, and N. A. Baharum, "A review on sample size determination for Cronbach's Alpha test: a simple guide for researchers," Malaysian Journal of Medical Sciences, vol. 25, no. 6, pp. 85-99, 2018.

[30] W. Deng, J. Xu, H. Zhao, and X. Z. Gao, "A novel gate resource allocation method using improved PSO-based QEA," IEEE Transactions on Intelligent Transportation Systems, 2020. 